\title{
Correction to: Paraneoplastic cerebellar degeneration as a presenting manifestation of non-Hodgkin's lymphoma
}

\author{
Satyan Nanda ${ }^{1} \cdot$ Rahul Handa $^{1} \cdot$ Atul Prasad $^{1} \cdot$ Rajiv Anand $^{1} \cdot$ Dhruv Zutshi ${ }^{1} \cdot$ Sujata K. Dass ${ }^{1} \cdot$ Prabhjeet Kaur Bedi $^{1}$. \\ Aarti Pahuja ${ }^{1}$. Pankaj Kumar Shah ${ }^{1} \cdot$ Bipan Sharma ${ }^{1} \cdot$ Nalin Malhotra $^{1}$
}

Published online: 8 March 2021

(C) Fondazione Società Italiana di Neurologia 2021

\section{Correction to: Neurological Sciences (2021) https://doi.org/10.1007/s10072-021-05046-z}

The original article contains an error. The article published with incorrect Abstract. The correct Abstract is presented here.

\begin{abstract}
Background: Paraneoplastic Cerebellar degeneration (PCD) is one of the classical paraneoplastic syndromes (PNS) which is characterised by subacute onset, progressive cerebellar ataxia and is usually associated with small cell lung carcinoma, adeno carcinoma of breast and ovary followed by Hodgkin's lymphoma.

Objective: We herein report a case of subacute onset, progressive cerebellar ataxia in a 37-year-old female, who on evaluation was found to have non-Hodgkin's lymphoma and experienced good clinical response to treatment.

Discussion: As compared to solid tumours, chances of association of PNS with Lymphomas is quite low and there are only few case reports in the literature showing association of PCD with non-Hodgkin's lymphoma. As PCD is one of the
\end{abstract}

classical PNS, it is very important to identify subtle cerebellar manifestations in an otherwise apparently normal individual, as early diagnosis and aggressive treatment can immensely improve the mortality and morbidity associated with this syndrome.

Conclusion: This case signifies the importance of suspecting PNS as an important differential diagnosis in a young patient presenting with subacute onset progressive cerebellar ataxia and evaluating her extensively for malignancy in spite of no paraneoplastic antibody been detected as early diagnosis and treatment can lead to gratifying response. We do agree that 2 weeks follow up is a short time interval to determine whether the response was sustained or not, for which a long term follow up is required.

The original article has been corrected.

Publisher's note Springer Nature remains neutral with regard to jurisdictional claims in published maps and institutional affiliations.
The online version of the original article can be found at https://doi.org/ 10.1007/s10072-021-05046-Z

Rahul Handa

rahulhanda0411@gmail.com

1 Department of Neurology, BLK Super Speciality Hospital, New Delhi, India 